PREPARED FOR THE U.S. DEPARTMENT OF ENERGY, UNDER CONTRACT DE-AC02-76CH03073

PPPL-3942

PPPL-3942

UC-70

\title{
Nonlinear Plasma Waves Excitation by Intense Ion Beams in Background Plasma
}

by

Igor D. Kaganovich, Edward A. Startsev, and Ronald C. Davidson

April 2004

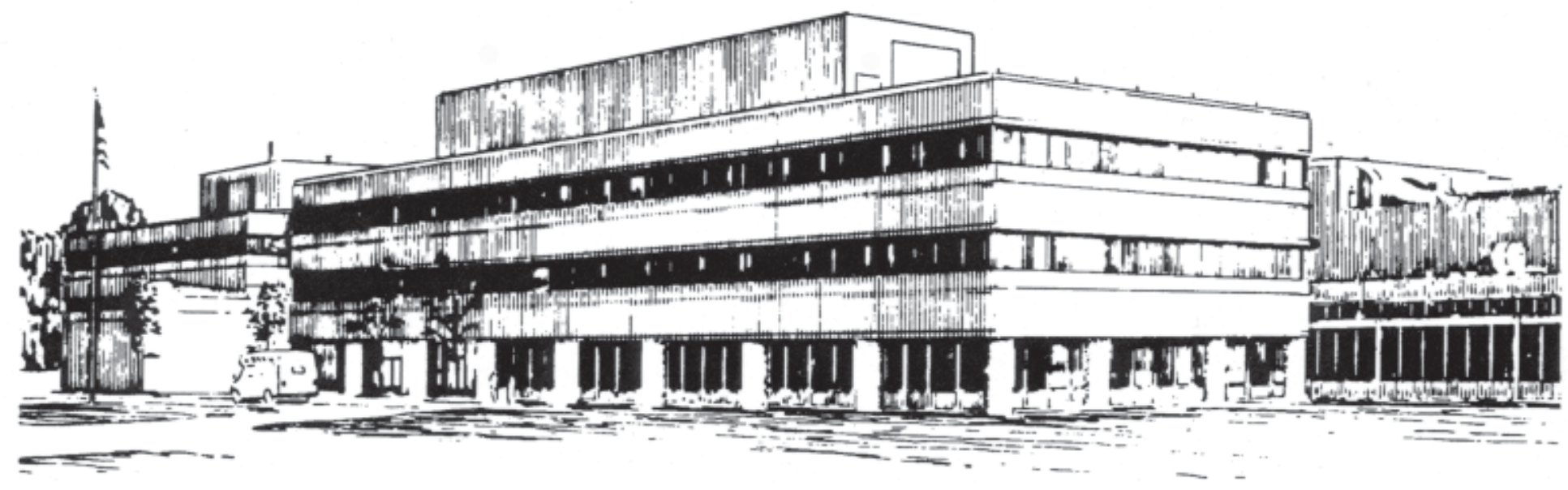

PRINCETON PLASMA PHYSICS LABORATORY PRINCETON UNIVERSITY, PRINCETON, NEW JERSEY 


\section{PPPL Reports Disclaimer}

This report was prepared as an account of work sponsored by an agency of the United States Government. Neither the United States Government nor any agency thereof, nor any of their employees, makes any warranty, express or implied, or assumes any legal liability or responsibility for the accuracy, completeness, or usefulness of any information, apparatus, product, or process disclosed, or represents that its use would not infringe privately owned rights. Reference herein to any specific commercial product, process, or service by trade name, trademark, manufacturer, or otherwise, does not necessarily constitute or imply its endorsement, recommendation, or favoring by the United States Government or any agency thereof. The views and opinions of authors expressed herein do not necessarily state or reflect those of the United States Government or any agency thereof.

\section{Availability}

This report is posted on the U.S. Department of Energy's Princeton Plasma Physics Laboratory Publications and Reports web site in Fiscal Year 2004. The home page for PPPL Reports and Publications is: http://www.pppl.gov/pub_report/

DOE and DOE Contractors can obtain copies of this report from:

U.S. Department of Energy

Office of Scientific and Technical Information

DOE Technical Information Services (DTIS)

P.O. Box 62

Oak Ridge, TN 37831

Telephone: (865) 576-8401

Fax: (865) 576-5728

Email: reports@adonis.osti.gov

This report is available to the general public from:

National Technical Information Service

U.S. Department of Commerce

5285 Port Royal Road

Springfield, VA 22161

Telephone: $1-800-553-6847$ or

(703) $605-6000$

Fax: (703) 321-8547

Internet: http://www.ntis.gov/ordering.htm 


\title{
Nonlinear plasma waves excitation by intense ion beams in background plasma
}

\author{
Igor D. Kaganovich, Edward A. Startsev, and Ronald C. Davidson \\ Plasma Physics Laboratory, Princeton University, Princeton, New Jersey 08543
}

Plasma neutralization of an intense ion pulse is of interest for many applications, including plasma lenses, heavy ion fusion, cosmic ray propagation, etc. An analytical electron fluid model has been developed to describe the plasma response to a propagating ion beam. The model predicts very good charge neutralization during quasi-steady-state propagation, provided the beam pulse duration $\tau_{b}$ is much longer than the electron plasma period $2 \pi / \omega_{p}$, where $\omega_{p}=\left(4 \pi e^{2} n_{p} / m\right)^{1 / 2}$ is the electron plasma frequency, and $n_{p}$ is the background plasma density. In the opposite limit, the beam pulse excites large-amplitude plasma waves. If the beam density is larger than the background plasma density, the plasma waves break. Theoretical predictions are compared with the results of calculations utilizing a particle-in-cell (PIC) code. The cold electron fluid results agree well with the PIC simulations for ion beam propagation through a background plasma. The reduced fluid description derived in this paper can provide an important benchmark for numerical codes and yield scaling relations for different beam and plasma parameters. The visualization of numerical simulation data shows complex collective phenomena during beam entry and exit from the plasma.

PAC numbes: 29.27.Bd, 29.27.Eg, 52.58.Hm, 52.65.Rr 


\section{Introduction}

Ion beam pulses are frequently used in many applications, including heavy ion inertial fusion ${ }^{1,2}$, positron beams for electron-positrons colliders ${ }^{3}$, high-density laserproduced proton beams for the fast ignition of inertial confinement fusion targets ${ }^{4}$, etc. In these applications unneutralized ballistic focusing is difficult due to the large repulsive space-charge force of the beam ions. To neutralize the ion beam charge, the ion beam can be transported through a background plasma. The plasma electrons tend to effectively neutralize the ion beam charge, and the background plasma can provide an ideal medium for ion beam focusing. There are many critical parameters for ion beam transport in a background plasma, including beam current, type of ion species, radial and longitudinal profiles of the beam density and plasma density, stripping and ionization cross sections of the beam ions and gas atoms, etc. This necessitates an extensive study for a wide range of parameters to determine the conditions for optimum beam propagation. To complement the numerical simulation studies, a number of reduced models have been developed ${ }^{5-9}$. Based on well-verified assumptions, reduced models can yield robust analytical and numerical descriptions and provide important scaling laws for the degrees of charge and current neutralization. Typically, the ion beam pulse propagation duration through the background plasma is long compared with the electron response time, which is

determined by the electron plasma frequency, $\omega_{p}=\left(4 \pi n_{p} e^{2} / m_{e}\right)^{1 / 2}$, where $n_{p}$ is the background plasma density. Therefore, after the beam passes through a short transition region, the plasma disturbances are stationary in the beam frame. The goal of this study is 
to develop a reduced nonlinear model, which describes the plasma disturbance excited by the intense ion beam pulse.

If the beam density is small compared to the background plasma density, a linear perturbation theory can describe well the plasma perturbations ${ }^{5}$. Here, we focus on the general case where the plasma density has an arbitrary value compared to the beam density. Reference 6 studied the transport of a long ion beam pulse utilizing the assumptions of complete current and charge neutralization. In the present study, we determine the degree of current and charge neutralization from a nonlinear analysis. Nonlinear fluid models have been developed for studies of the plasma response to a propagating laser pulse ${ }^{7}$. Considerable simplification of these models has been made by assuming that the plasma parameters depend on $z$ and $t$ exclusively through the combination $\zeta=z-c t$. Therefore, the solutions are time stationary in the laser pulse frame. Here, $z$ is the coordinate along the laser pulse propagation direction in the laboratory frame, $t$ is the time, and $c$ is the speed of light in vacuo. The reduced models are also based on the fact that the laser pulse moves with the speed of light. Plasma perturbations do not propagate faster than the speed of light. Therefore, different cross sections of the perturbed plasma perpendicular to the propagation direction do not communicate nonlocally with one another. As a result, a considerable reduction in the amount of necessary computation can be achieved, as will be explained in more detail below.

In the present study, a similar procedure has been developed for an ion beam pulse propagating through a background plasma. The ion beams under consideration are typically not relativistic. As a result, different cross sections perpendicular to the 
propagation direction do communicate nonlocally with one another via the Poisson equation: the electrostatic potential is a nonlocal function of the space charge over the entire beam pulse. However, for long beams with beam half-length much longer than the beam radius, $l_{b} \gg r_{b}$, only neighboring slices interact with one another. The assumption of long beams $\left(l_{b} \gg r_{b}\right)$ allows one to efficiently reduce the dimensionality of the equations. In recent calculations ${ }^{8,9}$, we studied the nonlinear quasi-equilibrium properties of an intense, long ion beam pulse propagating through a cold, background plasma, assuming that the beam pulse duration $\tau_{b}$ is much longer than the inverse electron plasma frequency, i.e., $\omega_{p} \tau_{b} \gg 1$. In the present study, we extend the previous results to general values of the parameter $\omega_{p} \tau_{b}$. The key assumption in this paper is that the electron thermal velocity can be neglected, because it is much smaller than ion beam velocity. This assumption allows us to use a fluid approximation and obtain an analytical solution for the self-electric and self-magnetic fields of the ion beam pulse.

\section{Basic equations for ion beam pulse propagation in background}

\section{plasma}

In many applications, the background plasma electrons are cold - the electron thermal velocity is small compared with the directed beam velocity ${ }^{10}$. Particle-in-cell simulations show that in most cases the electron flow is laminar and does not form multistreaming. For purposes of the plasma wave excitation study, we assume that the ion beam motion is unperturbed. Thus, the cold-fluid equations are used for the electron description, and thermal effects are neglected in the present study. The electron fluid equations together 
with Maxwell's equations comprise a complete system of equations describing the electron response to the propagating ion beam pulse. The electron cold-fluid equations consist of the continuity equation,

$\frac{\partial n_{e}}{\partial t}+\nabla \cdot\left(n_{e} \mathbf{V}_{e}\right)=0$

and the force balance equation,

$\frac{\partial \mathbf{p}_{e}}{\partial t}+\left(\mathbf{V}_{e} \cdot \nabla\right) \mathbf{p}_{e}=-e\left(\mathbf{E}+\frac{1}{c} \mathbf{V}_{e} \times \mathbf{B}\right)$,

where $-e$ is the electron charge, $\mathbf{V}_{\mathbf{e}}$ is the electron flow velocity, $\mathbf{p}_{e}=\gamma_{e} m_{e} \mathbf{V}_{e}$ is the average electron momentum, $m_{e}$ is the electron rest mass, and $\gamma_{e}=\left(1-\mathbf{V}_{e}^{2} / c^{2}\right)^{-1 / 2}$ is the relativistic mass factor. Maxwell's equations for the self-generated electric and magnetic fields, $\mathbf{E}$ and $\mathbf{B}$, are given by

$$
\begin{aligned}
& \nabla \times \mathbf{B}=\frac{4 \pi e}{c}\left(Z_{b} n_{b} \mathbf{V}_{b}-n_{e} \mathbf{V}_{e}\right)+\frac{1}{c} \frac{\partial \mathbf{E}}{\partial t}, \\
& \nabla \times \mathbf{E}=-\frac{1}{c} \frac{\partial \mathbf{B}}{\partial t},
\end{aligned}
$$

where $\mathbf{V}_{\mathbf{b}}=$ const is the ion beam flow velocity, $n_{e}$ and $n_{b}$ are the number densities of the plasma electrons and beam ions, respectively (far away from the beam $n_{e} \rightarrow n_{p}$, where $n_{p}$ is background plasma density), and $Z_{b}$ is the ion beam charge state. The plasma ions are pushed away from the ion beam pulse by the radial electric field. However, if the ion beam pulse duration is sufficiently short $\left(2 l_{b}<r_{b} \sqrt{M / m_{e}}\right.$, where $M$ is the plasma ion 
mass), the plasma ions do not have time to move outside the beam radius, and they can be assumed to remain stationary with $\boldsymbol{V}_{\boldsymbol{i}}=0$ and $n_{i}=n_{p}$.

Additional simplification is achieved by applying the conservation law of generalized vorticity $\Omega$, where

$\Omega \equiv \nabla \times \mathbf{p}_{e}-\frac{e}{c} \mathbf{B}$

In fluid mechanics, for incompressible flow, the vorticity $\nabla \times \mathbf{V}$ is conserved along the path of a fluid element. Moreover, if all the streamlines originate from the region where the vorticity is equal to zero, then the vorticity is equal to zero everywhere, and the flow is eddy-free. In the background plasma without the beam pulse, $\mathbf{p}_{e}=\mathbf{B}=0$ and $\Omega=\mathbf{0}$. Therefore, it follows from the conservation of generalized vorticity, similar to fluid mechanics, that $\Omega$ is equal to zero in the beam pulse as well, i.e.,

$\nabla \times \mathbf{p}_{e}=\frac{e}{c} \mathbf{B}$

Note that Eq. (6) is a consequence of the fluid description and is not an priori assumption. Substituting Eq.(6) into Eq.(2) yields

$\frac{\partial \mathbf{p}_{e}}{\partial t}+\nabla K_{e}=-e \mathbf{E}$

where $K_{e}=\left(\gamma_{e}-1\right) m_{e} c^{2}$ is the electron kinetic energy. Note that the inertia terms in Eq.(7) are comparable in size to the Lorentz force term and cannot be omitted. Estimating the self-magnetic field from Eq. (7), we conclude that the electron gyroradius is of order the beam radius. This is a consequence of the fact that the electrons originate from the region of zero magnetic field in front of the beam. If most electrons are dragged along 
with the beam and originate from the region of large magnetic field, the situation may be different ${ }^{11,12}$.

\section{Approximate system of equations for long charge bunches $\left(l_{b} \gg r_{b}\right)$}

In this section, we assume a long ion beam pulse $\left(l_{b} \gg r_{b}\right)$, but relax the assumption of a dense plasma used in Refs.8 and 9, i.e., the condition $V_{b} / \omega_{p}<<l_{b}$. The typical longitudinal scale of electron density perturbations is $V_{b} / \omega_{p}$. If $V_{b} / \omega_{p} \ll r_{b}$, the main spatial variations are in the longitudinal direction and the electron dynamics can be described by a one-dimensional model. In the opposite case, when $V_{b} / \omega_{p}>>r_{b}$, the main spatial variations are in the transverse direction, and the longitudinal derivatives can be neglected in comparison with the radial derivatives in Poisson's equation. The criterion $V_{b} / \omega_{p}>>r_{b}$ can be expressed as a condition on the total ion beam current, $I_{b}<17 \beta^{3} n_{b} / n_{p} k A$, and this condition pertains to ion beams that aren't extremely intense. We also assume an axisymmetric beam pulse $(\partial / \partial \theta=0)$. The dependence on $z$ and $t$ is assumed to be exclusively through $\zeta=z-V_{b} t$. Therefore solutions are time stationary in the beam frame. This gives

$$
\begin{aligned}
& \left(V_{e z}-V_{b}\right) \frac{\partial n_{e}}{\partial \zeta}+\frac{1}{r^{n}} \frac{\partial}{\partial r}\left(r^{n} n_{e} V_{e r}\right)=0, \\
& \frac{1}{r^{n}} \frac{\partial}{\partial r}\left(r^{n} E_{r}\right)=4 \pi e\left(Z_{b} n_{b}+n_{p}-n_{e}\right), \\
& \left(V_{e z}-V_{b}\right) \frac{\partial}{\partial \zeta} p_{e r}+\frac{\partial K_{e}}{\partial r}=-e E_{r} .
\end{aligned}
$$


Here, $n=0$ for slab geometry $(r \rightarrow x)$ and $n=1$ for a cylindrical beam. Singlecharged plasma ions are assumed with $Z_{b}=1$. As a result, the two-dimensional problem is reduced to a one-dimensional problem with time derivatives being replaced by $\left(V_{e z}-V_{b}\right) \partial / \partial \zeta$. It follows from Eq.(6) and the assumption of a long charge bunch that the azimuthal self-magnetic field for $\partial / \partial \theta=0$ is determined in terms of the longitudinal flow velocity, which gives

$$
\begin{aligned}
& B_{\theta}=-\frac{c}{e} \frac{\partial p_{e z}}{\partial r} \\
& -\frac{1}{r} \frac{\partial}{\partial r} r \frac{\partial}{\partial r} p_{e z}=\frac{4 \pi e}{c}\left(Z_{b} n_{b} V_{b}-n_{e} V_{e z}\right) .
\end{aligned}
$$

Equation (12) determines the longitudinal electron flow velocity and can be used to calculate the degree of current neutralization of the beam. In Eq. (12), we have neglected the displacement current ${ }^{13}$. The electric field along the beam propagation direction can be determined from Eq. (7), which gives

$$
\left(V_{e z}-V_{b}\right) \frac{\partial}{\partial \zeta} p_{e z}+\frac{\partial K_{e}}{\partial \zeta}=-e E_{z} .
$$

It follows from Eqs.(10) and (13) that $E_{z} / E_{r} \sim r_{b} / l_{b} \ll 1$. Therefore, the longitudinal electric field is small compared with the radial electric field, and $E_{z}$ has been neglected in comparison with $E_{r}$ in Poisson's equation (9).

To check the theoretical predictions, we have utilized a two-dimensional (2D) electromagnetic particle-in-cell (PIC) code, called edPIC ${ }^{14}$. The code uses a leap-frog, finite-difference scheme ${ }^{15}$ to solve Maxwell's equations (3) and (4) on a planar, twodimensional rectangular grid in the laboratory frame. EdPIC uses a moving-window 
approach. The window of the simulations is shifted after a few time steps so that the window is moving with the beam on average. The current deposition scheme is designed to conserve charge exactly ${ }^{16}$, so there is no need to solve Poisson's equation. Since the plasma ahead of the pulse is electrically neutral, the boundary conditions for the fields on the front boundary of the simulation box are trivial $(\mathbf{E}=\mathbf{B}=0)$. The boundaries are located at $3 r_{b}$ and $1.5 l_{b}$ in the radial and axial directions, respectively. As can be seen from Fig.1, the plasma and field perturbations do not reach the boundaries (as the boundaries are located far away from the beam at distances larger than the beam radius and the skin depth). In the present simulations, the dynamics of the (stationary) background ions is neglected, and the background plasma electrons are initially cold. The beam ions are represented by a stationary (in the moving frame) current density on the simulation grid. To advance the electrons, we use the time-centered, leap-frog scheme first introduced in Ref. 17. Various simulations obtained using the edPIC particle-in-cell code are presented in Refs. 8, 18 and 19. The fluid simulations utilize the MacCormack finite-difference scheme $^{20}$ to solve the system of equations (8) - (12) on a two-dimensional rectangular grid.

In Fig. 1, we present a detailed comparison of the fluid and PIC results in slab geometry. The fluid simulation results agree well with the results of the two-dimensional electromagnetic PIC simulations. Figure 1 shows similar beams with a constant ratio of beam length to radius $\left(l_{b} / r_{b}=10\right)$ and different beam densities. The background plasma density is maintained at twice the beam density. If the plasma density is sufficiently small that the plasma period is long compared with the beam pulse duration, i.e., $2 \pi / \omega_{p}>2 l_{b} / V_{b}$, the plasma does not have time to respond to the beam pulse and a 
plasma wake appears behind the beam pulse, as evident in Fig.1(a). As the beam and background plasma density increase, the plasma period shortens and the number of plasma oscillations increases during the beam pulse. Notwithstanding the fact that $n_{b}=0.5 n_{p}$, the plasma oscillations excited by the beam pulse are quite large - the electron density perturbation can be as large as $3 n_{p}$ [see Fig. 1(b)].

Note that the longitudinal scale of the plasma oscillations is of order $V_{b} / \omega_{p}$ and can be smaller than the beam radius $\left(V_{b} / \omega_{p}<r_{b}\right)$ as in Fig. 1(d). As a result, the assumption of mainly radial dynamics fails in this case, and the agreement between the fluid and particle-in-cell simulations is not as good as in the case where $V_{b} / \omega_{p}>r_{b}$. However, if the plasma oscillations are not large-amplitude, i.e., the perturbations in the electron density are small compared with the background density, which corresponds to $Z_{b} n_{b} \ll n_{p}$, the plasma oscillations can be described by linear theory. Interestingly, the reduced system of equations (8) - (12) recovers the results of the full system of equations in the linear approximation. Indeed, in the linear case, when $Z_{b} n_{b} \ll n_{p}$, the equation for the plasma oscillations is

$$
V_{b}^{2} \frac{\partial^{2}}{\partial \zeta^{2}} n_{e}+\omega_{p}^{2}\left(n_{e}-Z_{b} n_{b}-n_{p}\right)=0
$$

Equation (14) is readily derived from the linearized version of Eqs.(1) and (2) and Poisson's equation, and is not restricted by any requirements on the beam radius. It can also be derived from the reduced system of equations (8) - (12), if the nonlinear terms are neglected. Because the reduced model consisting of the system of equations (8) - (12) gives the same results in the limit $V_{b} / \omega_{p}>>r_{b}$, and in the linear case $Z_{b} n_{b} \ll n_{p}$ for 
any values of $V_{b} / \omega_{p}$ and $r_{b}$, it also applies reasonably well in the intermediate case where $V_{b} / \omega_{p} \sim r_{b}$, as can be seen in Fig.1(d).

Figure 1(c) shows clearly the limitations of the fluid approach. Pairs of fluid electron trajectories intersect at the points $x= \pm 0.19 c / \omega_{p}$ and $y=-1.8 c / \omega_{p}$. Correspondingly, the fluid equations have a singularity at this point, and the electron density tends to infinity [see Fig. 1(c), bottom frame]. Particle-in-cell simulations do not show any singularity in the electron density as evident in Fig. 1(c) [top left and bottom frames]. Therefore, the presence of the singularity in the fluid solution for the electron density points to a failure of the fluid model. Specifically, the basic assumption of the fluid approach that there is a uniquely defined, single-valued electron flow velocity at every point breaks down.

Figure 2 shows the total current and electric field in the case of a long beam pulse. Because the beam pulse length, $2 l_{b} / V_{b}$, is much longer than the plasma period, $2 \pi / \omega_{p}$, ( $l_{b} \omega_{p} / \pi V_{b}=9.6$ ), the ion beam charge is well neutralized. However, the ion density rises steeply at the head of the beam pulse on a time scale comparable with the plasma period. This sudden increase in ion density drives many plasma oscillations during the beam pulse. Because the ion beam charge density is well neutralized in an average sense, the total current (the sum of the ion beam current and the electron current) is divergence free $(\nabla \cdot \mathbf{j}=0)$. This means that the ion beam current should be short-circuited outside the beam pulse by the electron return current, which is illustrated in Fig. 2(a). In Fig.2 (b), we note that the longitudinal electric field is small compared with the radial electric field 
( $E_{z} / E_{r} \sim r_{b} / l_{b}$ ). Short wavelength plasma oscillations at the beam pulse head, however, can produce larger longitudinal electric fields.

The fluid approach may not be applicable for a tenuous background plasma. In Fig.1(c), the intersecting fluid electron trajectories originate from different radial positions ahead of the beam pulse. In the limit $n_{b}>n_{p}$, the intersecting electron trajectories can originate from different longitudinal positions ahead of the beam pulse, which corresponds to plasma wave breaking in one-dimensional geometry as illustrated in Fig. 3. If the beam density is less than the background plasma density $\left(n_{b}<n_{p}\right)$, the amplitude of the plasma oscillations is finite. In Fig.3(a), the electron dynamics can be described by the fluid approach: a phase space plot shows that there is a uniquelydefined, single-valued electron flow velocity at every point. If $n_{b}>n_{p}$, the electron density oscillations may become large, and the intersecting electron trajectories originate from different longitudinal positions. This is evident from the phase space plot shown in Figs. 3(b) and 3(c). The time evolution in phase space of the electron distribution corresponds to the complex process of electron heating due to plasma wave breaking. The analysis described above shows that for good charge neutralization the ion beam should be neutralized by plasma with density larger than the ion beam density. In the opposite case, the ion beam head may excite large-amplitude plasma waves. This will lead to electron heating and the generation of large, uncontrollable radial electric fields, which may be detrimental to ion beam focusing. 


\section{Conclusions}

We have shown that the electron perturbations excited by a long ion beam pulse $\left(l_{b} \gg r_{b}\right)$ can be well described in the fluid approximation by the reduced system of equations (8) - (12). Based on well-verified assumptions, this reduced model can yield robust analytical and numerical descriptions and provide important scaling laws for the degrees of charge and current neutralization. However, the utilization of the cold-fluid model may be limited by the wave-breaking condition (intersection of fluid electron trajectories), especially in the case $n_{b}>n_{p}$.

The approach used here can be generalized to the case of a nonuniform, nonstationary, warm electron fluid. This research is now underway. This more general approach will be especially useful for studies of the long-time evolution of ion beam pulses on time scales much longer than the plasma period $2 \pi / \omega_{p}$, because it enables one to eliminate the fast processes (at the plasma frequency) from the governing equations, thereby, substantially reducing the computational requirements. For example, simplified beam envelope equations can be developed based on the reduced system of equations. These envelope equations are known to predict beam focusing with reasonable $\operatorname{accuracy}^{21}$.

The analysis described in this paper shows that for good charge neutralization the ion beam should be neutralized by plasma with density larger than the ion beam density. The condition for avoiding large-amplitude plasma wave generation requires that the rise time of the ion beam pulse be much longer than the plasma period $2 \pi / \omega_{p}$. In the opposite case, the ion beam head excites large-amplitude plasma waves. This may lead to 
electron heating and the generation of large, uncontrollable radial electric fields, which could be detrimental to ion beam focusing. The parameter which controls the degree of current neutralization is the ratio of the skin depth to the beam radius and is discussed in greater detail elsewhere ${ }^{8,9}$.

Finally, although steady-state propagation of the ion beam pulse in a background plasma has been well studied, the beam entry and exit from the plasma requires additional research. We have produced movies that illustrate the complex collective phenomena that occur during beam entry into and exit from the plasma ${ }^{22}$. Additional discussion of this subject is also provided in Ref. 19.

Acknowledgments: This research was supported by the U.S. Department of Energy Office of Fusion Energy Sciences and the Office of High Energy Physics. 


\section{Figure Captions}

Figure 1. Neutralization of an ion beam pulse during steady-state propagation of the beam pulse through a cold, uniform, background plasma in planar geometry. The figure shows comparisons between the PIC simulations and the fluid description. The beam propagates in the y-direction. The beam density has a flat-top density profile, and the contour (red in color) lines show the beam pulse edge. Shown in the figure are color plots of the normalized electron density $\left(n_{e} / n_{p}\right)$ for particle-in-cell simulations (top left) and the fluid model consisting of Eqs.(8) - (12) (top right) in $\left(x \omega_{p} / c, y \omega_{p} / c\right)$ space. The lower figure shows the normalized electron density $\left(n_{e} / n_{p}\right)$, and the normalized longitudinal current $\left(j_{y} / e n_{p} c\right)$ in the beam cross-section at $x=0$ (lowest curves). The (brown in color) contours in the upper figure show the electron trajectories in the beam frame. The beam velocity is $V_{b}=0.5 c$, the beam density is $n_{b}=0.5 n_{p}$, and the ion beam charge state is $Z_{b}=1$. The beam dimensions correspond to $l_{b} / r_{b}=10$ and (a) $l_{b}=0.3 c / \omega_{p}$, (b) $l_{b}=1.0 c / \omega_{p}$, (c) $l_{b}=3.0 c / \omega_{p}$, and (d) $l_{b}=10 c / \omega_{p}$. The dashed lines in the lower figure 1 (c) show wave breaking at $\mathrm{x}=0.19 c / \omega_{p}$.

Figure 2. The excitation of plasma waves by the beam head is calculated in twodimensional slab geometry using the edPIC code for the following beam parameters: $V_{b}=0.5 c, Z_{b}=1, n_{b}=n_{p}, l_{b} / r_{b}=10$ and $l_{b}=15 c / \omega_{p}$. Shown in the figure are electron charge density contour plots in $\left(x \omega_{p} / c, y \omega_{p} / c\right)$ space and the vector fields for (a) the total current, and (b) minus the electric field, $-E$. 
Figure 3. Electron phase space for 1D PIC simulations of the beam entering the plasma at $t=0$. Here, $V_{b}=0.5 c, Z_{b}=1, l_{b}=7.5 \mathrm{c} / \omega_{p}$ and (a) $n_{b}=n_{p}$, (b) $n_{b}=2 n_{p}$, and (c) $n_{b}=2 n_{p}$. The steady state establishes after the beam enters the plasma in case (a). Under these circumstances, the plasma phase space also shows applicability of the fluid approximation. However, in case (b) the plasma wave breaks and the electrons are heated by wave-particle interactions. In this situation, the cold-fluid approximation is not applicable. Moreover, the phase space slowly evolves with time as shown. The times after entering the plasma correspond to (b) $t=113 / \omega_{p}$, and (c) $t=245 / \omega_{\mathrm{p}}$. 
${ }^{1}$ B.G. Logan, C.M. Celata, J. Kwan, E.P. Lee, M. Leitner, P.A. Seidl, S.S. Yu, J.J.

Barnard, A. Friedman, W.R. Meier, R.C. Davidson, Laser and Particle Beams 20, 369 (2002);

C.M. Celata, F.M. Bieniosek, E. Henestroza, J.W. Kwan, E.P. Lee, G. Logan, L. Prost, P.A. Seidl, J.L. Vay, W.L. Waldron, S.S. Yu, J.J. Barnard, D.A. Callahan, R.H. Cohen, A. Friedman, D.P. Grote, S.M. Lund, A. Molvik, W.M. Sharp, G. Westenskow, R.C. Davidson, P. Efthimion, E. Gilson, L.R. Grisham, I. Kaganovich, H. Qin, E.A. Startsev, S. Bernal, Y. Cui, D. Feldman, T.F. Godlove, I. Haber, J. Harris, R.A. Kishek, H. Li, P.G. O'Shea, B. Quinn, M. Reiser, A. Valfells, M. Walter, Y. Zou, D.V. Rose, D.R. Welch Phys. Plasmas 10, 2063 (2003);

S.S. Yu, W.R. Meier, R.P. Abbott, J.J. Barnard, T. Brown, D.A. Callahan, C. Debonnel, P. Heitzenroeder, J.F. Latkowski, B.G. Logan, S.J. Pemberton, P.F. Peterson, D.V. Rose, G.L. Sabbi, W.M. Sharp, D.R. Welch, Fusion Science \& Technology 44, 266 (2003).

${ }^{2}$ W.M. Sharp, D.A. Callahan, M. Tabak, S.S. Yu, P.F. Peterson, D.R. Welch, D.V. Rose, C.L. Olson, Fusion Science \& Technology 43, 393 (2003).

${ }^{3}$ M.J. Hogan, C.E. Clayton, C. Huang, P. Muggli, S. Wang, B.E. Blue, D. Walz, K.A. Marsh, C.L. O'Connell, S. Lee, R. Iverson, F.J. Decker, P. Raimondi, W.B. Mori, T.C. Katsouleas, C. Joshi, R.H. Siemann, Phys. Rev. Lett. 90, 205002 (2003).

${ }^{4}$ K.Krushelnick, E.L. Clark, R. Allott, F.N. Beg, C.N. Danson, A. Machacek, V. Malka, Z. Najmudin, D. Neely, P.A. Norreys, M.R. Salvati, M.I.K. Santala, M. Tatarakis, I. Watts, M. Zepf, A.E. Dangor, IEEE Trans. Plasma Sci. 28, 1184 (2000). 
${ }^{5}$ P. Chen, J.M. Dawson, R. W. Huff and T. Katsouleas, Phys. Rev. Lett. 51, 693 (1985).

${ }^{6}$ K. Hahn and E. P. Lee, J. Fusion Engineering and Design 32-33, 417 (1996).

${ }^{7}$ P. Mora and T.M. Antonsen, Phys. Plasmas 4, 217 (1997).

${ }^{8}$ I. D. Kaganovich, G. Shvets, E. Startsev and R. C. Davidson, Physics of Plasmas 8, 4180 (2001).

${ }^{9}$ I. D. Kaganovich, E. Startsev and R. C. Davidson, Laser and Particle Beams 20, 497 (2002).

${ }^{10}$ Here, we consider typical ion beams for a heavy ion fusion driver with velocity $V_{b} \sim 0.2 c$, or relativistic proton or positron beams with $V_{b} \simeq c$. The electron temperature is typically a few $\mathrm{eV}$ in the background plasmas produced by conventional plasma sources. This gives an electron thermal velocity of order $0.004 \mathrm{c}$ ( $\left.T_{e}=4 \mathrm{eV}\right)$. Therefore, the electron thermal velocity is 50 times smaller than the ion beam velocity, and unless the plasma density is 50 times larger than the ion beam density, the electron thermal velocity is much smaller than ion beam velocity.

${ }^{11}$ M. Rosenbluth, E.P. Lee and R. Briggs, private communication (2001).

${ }^{12}$ D. R. Welch, D. V. Rose, B. V. Oliver, T. C. Genoni, R. E. Clark, C. L. Olson and S. S. Yu, Phys. Plasmas 9, 2344 (2002); D. R. Welch, D. V. Rose, W.M. Sharp, C. L. Olson and S. S. Yu, Laser and Particle Beams 20, 621 (2002).

${ }^{13}$ If $V_{b} / \omega_{p} \ll l_{b}$, the displacement current is small compared with the electron current and can be neglected. If $V_{b} / \omega_{p} \gg l_{b}$, the plasma does not have time to respond to the ion beam pulse, and both the electron current and the displacement current are small compared to the ion beam current and can be neglected. If $V_{b} / \omega_{p} \sim l_{b}$, then 
$c / \omega_{p} \sim r_{b}\left(l_{b} / r_{b}\right) \beta \gg r_{b}$, and the skin depth is larger than the beam radius [for example, see Fig.3(c)]. In this case the ion beam current is not neutralized: both the electron current and the displacement current are small compared to ion beam current and can be neglected. As a result, the displacement current can be neglected for all cases in Eq.(12).

${ }^{14}$ Startsev, E. A. and McKinstrie, C. J., Phys. Plasmas, 10, 2552 (2003).

${ }^{15}$ C.K. Birdsall and A. B. Langdon, Plasma Physics via Computer Simulations, 2nd Ed., (Plenum, New York, 1984).

${ }^{16}$ R. L. Morse and C. W. Nielson, Phys. Fluids 14, 830 (1970).

${ }^{17}$ J. P. Boris and D. A. Hammer, Phys. Rev. E 10, 3338 (1969).

${ }^{18}$ I. D. Kaganovich, E. Startsev, S. Klasky and R. C. Davidson, Special Issue on Images in Plasma Science, IEEE Trans. Plasma Science 30, 12 (2002).

${ }^{19}$ I. D. Kaganovich, E. Startsev and R. C. Davidson, "Analytical and numerical studies of the complex interaction of a fast ion beam pulse with a background plasma”, to be published in Physica Scripta (2004).

${ }^{20}$ R.B. Horne and M. P. Freeman, J. Comp. Physics 171, 182 (2001).

${ }^{21}$ R. Govil, W.P. Leemans, E.Yu. Backhaus and J.S. Wurtele, Phys. Rev. Lett. 83, 3202 (1999).

${ }^{22}$ Movies show highly complex collective behavior of the electron motion during the entry of the intense beam into the background plasma. The simulations have been performed using two dimensional edPIC code in slab geometry. The beam propagates in the y-direction. The beam velocity is half speed of light.

Movie 1. (file 7_den.avi) Shown in the movie are color plots of the normalized electron density (normalized on the background plasma density) obtained in particle-in-cell simulations. The beam density is half of background density. The beam pulse dimensions are the beam radius is equal to 1.5 of the skin depth and half length is 7.5 of the skin depth. The beam density profile is flattop with smooth edges at $20 \%$ of the beam size. 
Movie 2. (file 7_jx.avi) The same as previous movie but shows color plots of the normalized total current in y direction. Current is normalized on the background plasma density times electron charge and speed of light.

Movie 3 (file 30_den.AVI) The same as movie 1 but for a more intense ion beam. The beam density is five times of the background density; the beam pulse dimensions are the beam radius is 1.5 of the skin depth and half length is 30 of the skin depth.

Movie 3 shows the development of hose-like structures and electron holes during the beam entry, which are absent in movies 1 and 2. Movies 1 and 2 show the excitation of plasma waves during beam exit from the plasma. In contrast to the steady-state propagation, where the plasma waves establish a stationary stripe-like pattern after the beam exits the plasma, the plasma waves form a nonstationary periodic pattern, resembling butterfly-wing motion. 
(a)
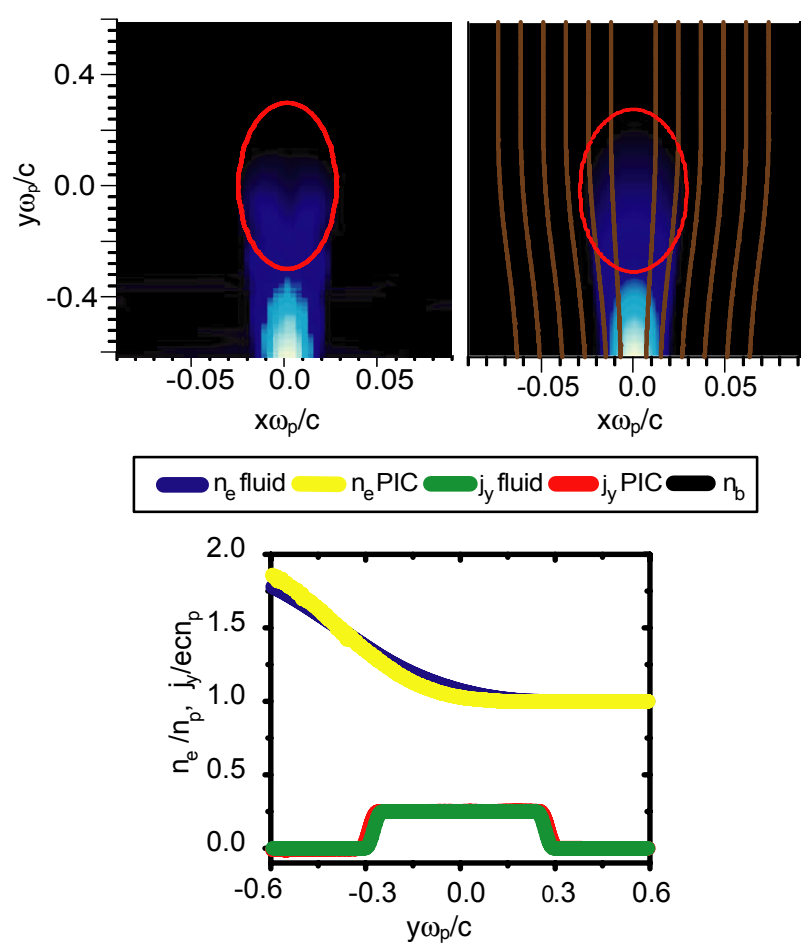

(c)
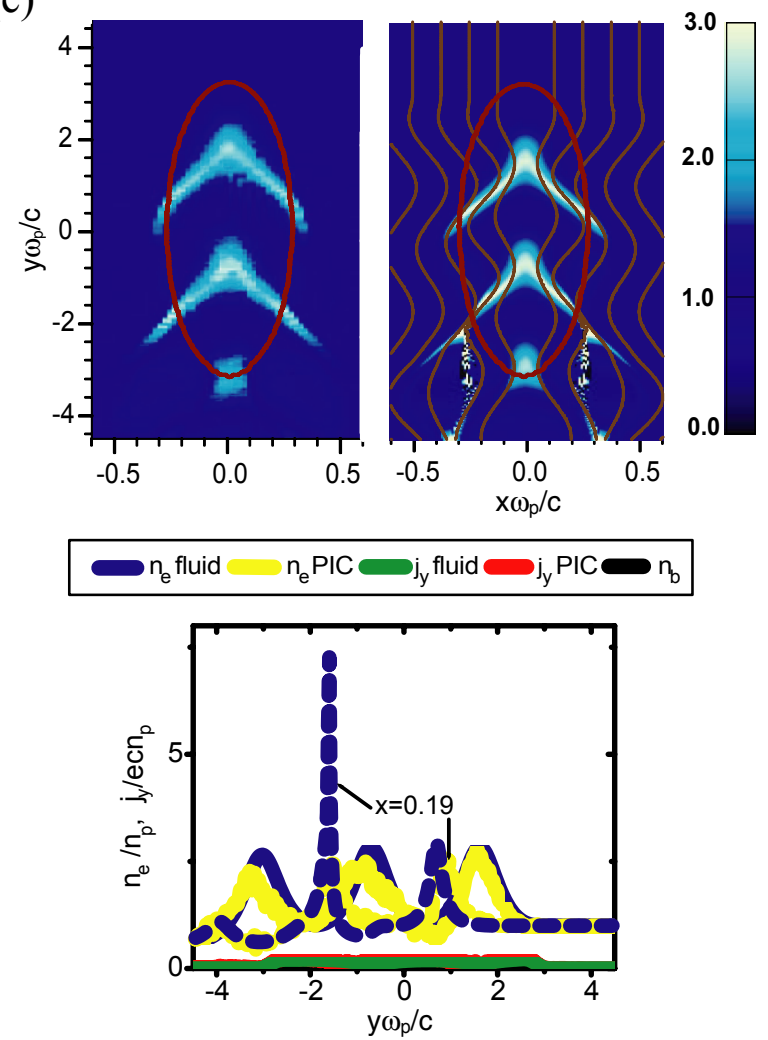
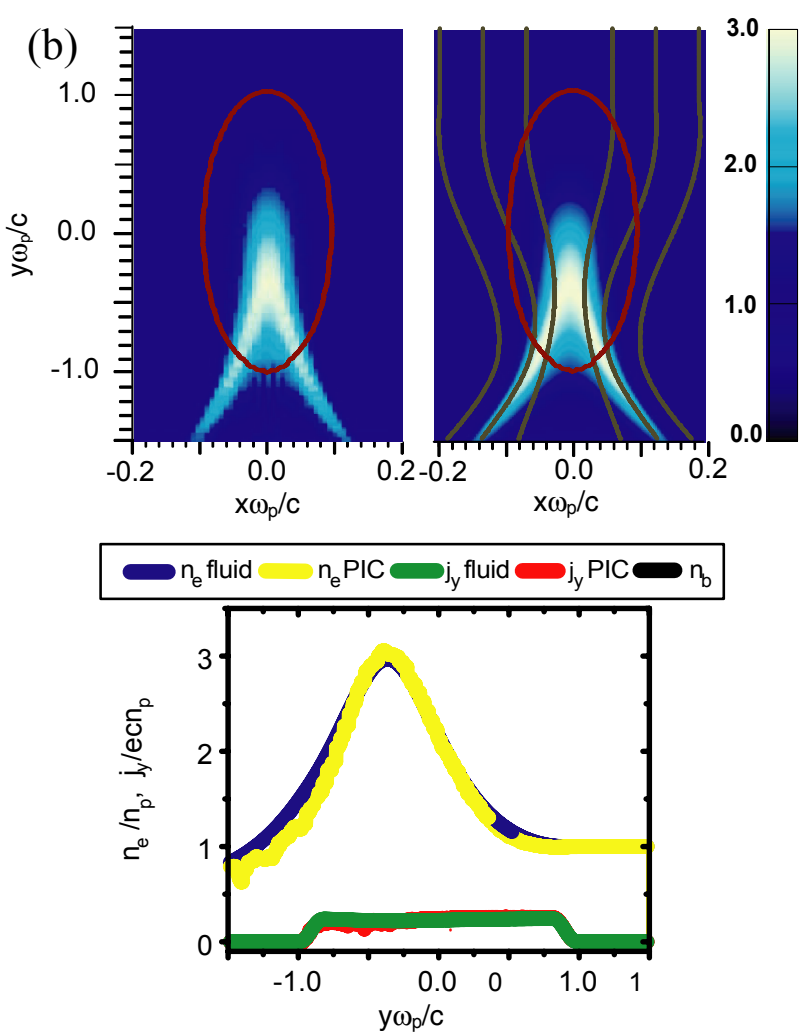

(d)
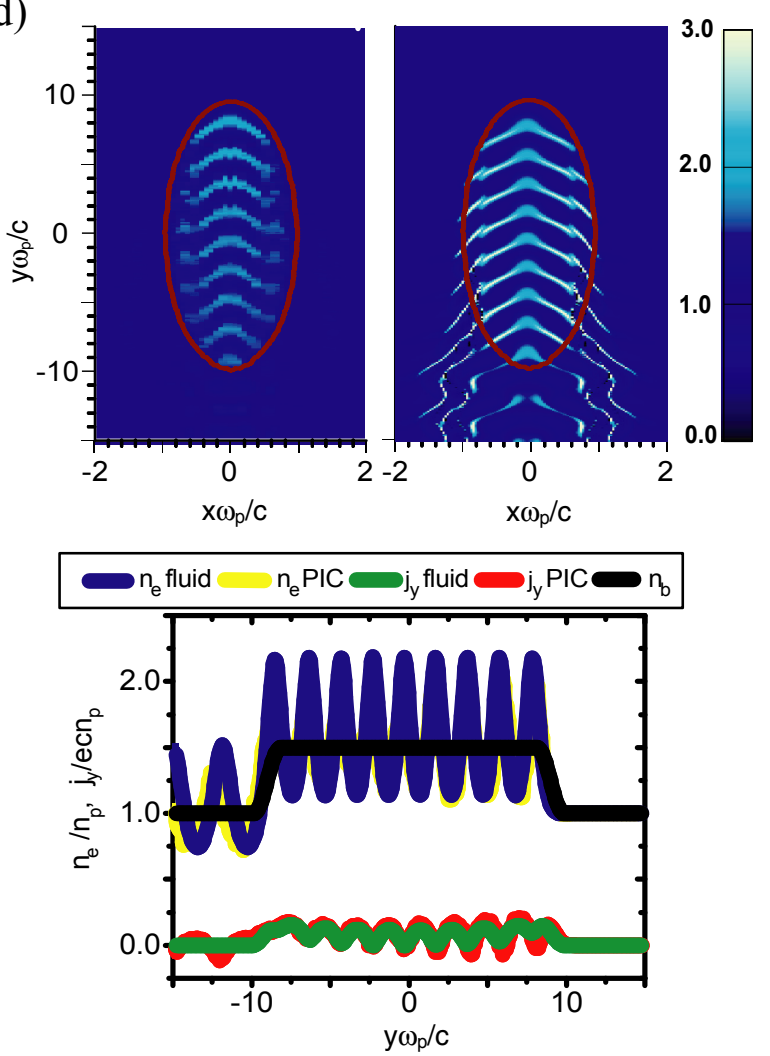
(a)

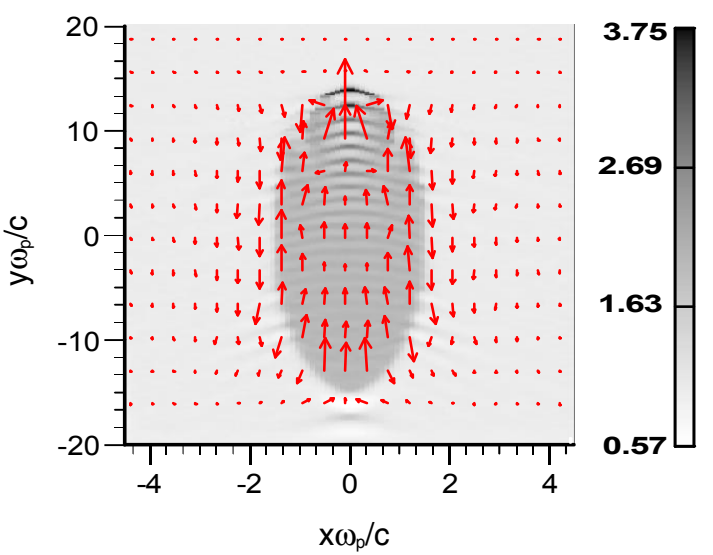

(b)

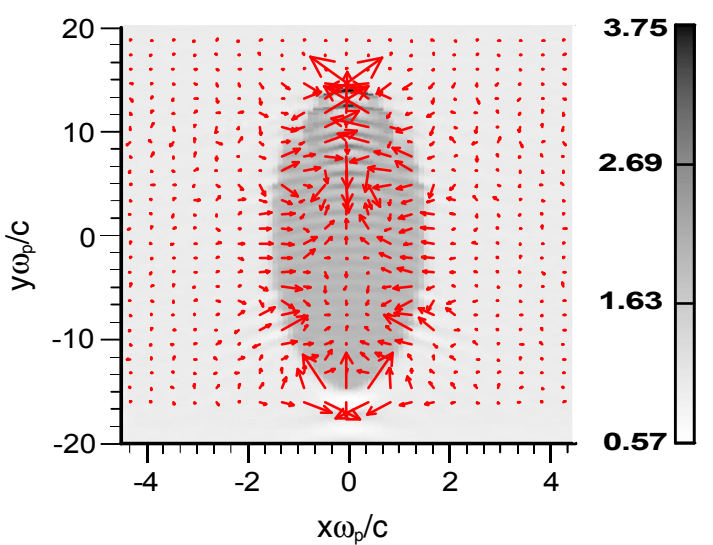



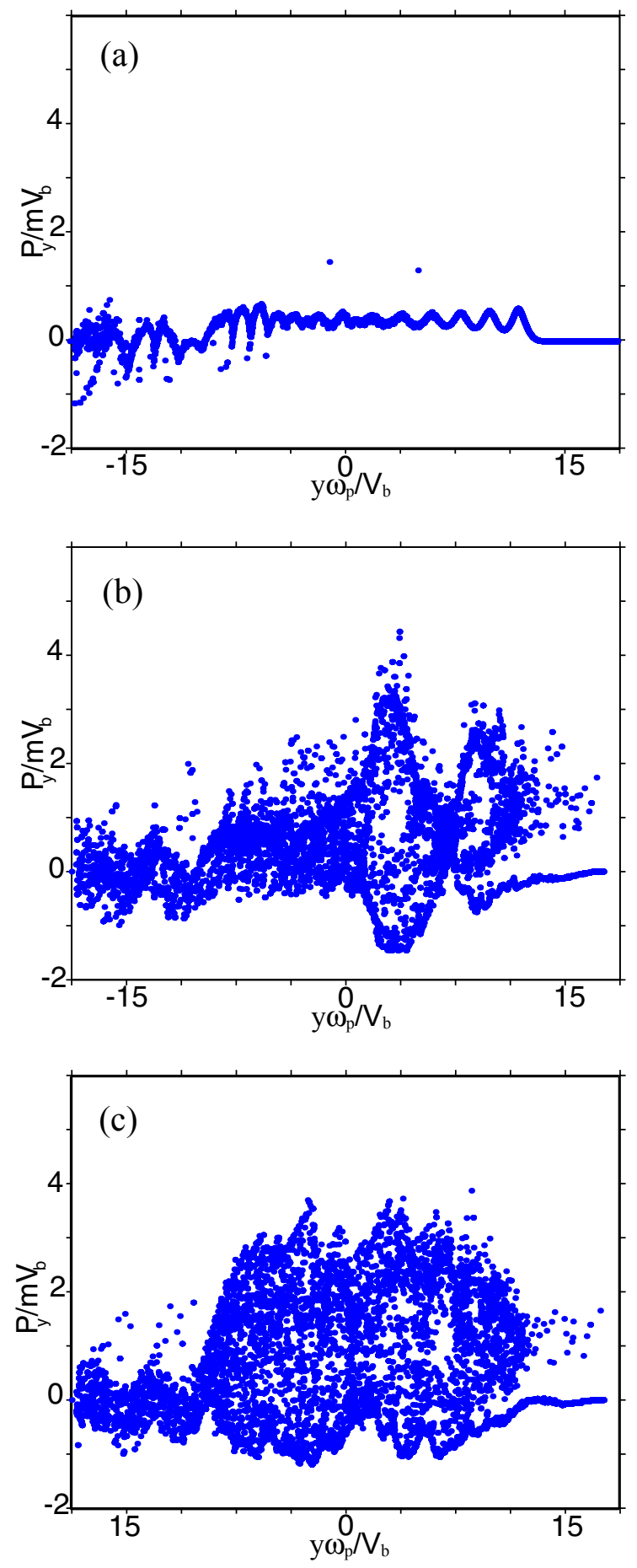


\section{External Distribution}

Plasma Research Laboratory, Australian National University, Australia

Professor I.R. Jones, Flinders University, Australia

Professor João Canalle, Instituto de Fisica DEQ/IF - UERJ, Brazil

Mr. Gerson O. Ludwig, Instituto Nacional de Pesquisas, Brazil

Dr. P.H. Sakanaka, Instituto Fisica, Brazil

The Librarian, Culham Laboratory, England

Mrs. S.A. Hutchinson, JET Library, England

Professor M.N. Bussac, Ecole Polytechnique, France

Librarian, Max-Planck-Institut für Plasmaphysik, Germany

Jolan Moldvai, Reports Library, Hungarian Academy of Sciences, Central Research Institute for Physics, Hungary

Dr. P. Kaw, Institute for Plasma Research, India

Ms. P.J. Pathak, Librarian, Institute for Plasma Research, India

Ms. Clelia De Palo, Associazione EURATOM-ENEA, Italy

Dr. G. Grosso, Instituto di Fisica del Plasma, Italy

Librarian, Naka Fusion Research Establishment, JAERI, Japan

Library, Laboratory for Complex Energy Processes, Institute for Advanced Study, Kyoto University, Japan

Research Information Center, National Institute for Fusion Science, Japan

Dr. O. Mitarai, Kyushu Tokai University, Japan

Dr. Jiangang Li, Institute of Plasma Physics, Chinese Academy of Sciences, People's Republic of China

Professor Yuping Huo, School of Physical Science and Technology, People's Republic of China

Library, Academia Sinica, Institute of Plasma Physics, People's Republic of China

Librarian, Institute of Physics, Chinese Academy of Sciences, People's Republic of China

Dr. S. Mirnov, TRINITI, Troitsk, Russian Federation, Russia

Dr. V.S. Strelkov, Kurchatov Institute, Russian Federation, Russia

Professor Peter Lukac, Katedra Fyziky Plazmy MFF UK, Mlynska dolina F-2, Komenskeho Univerzita, SK-842 15 Bratislava, Slovakia

Dr. G.S. Lee, Korea Basic Science Institute, South Korea

Institute for Plasma Research, University of Maryland, USA

Librarian, Fusion Energy Division, Oak Ridge National Laboratory, USA

Librarian, Institute of Fusion Studies, University of Texas, USA

Librarian, Magnetic Fusion Program, Lawrence Livermore National Laboratory, USA

Library, General Atomics, USA

Plasma Physics Group, Fusion Energy Research Program, University of California at San Diego, USA

Plasma Physics Library, Columbia University, USA

Alkesh Punjabi, Center for Fusion Research and Training, Hampton University, USA

Dr. W.M. Stacey, Fusion Research Center, Georgia Institute of Technology, USA

Dr. John Willis, U.S. Department of Energy, Office of Fusion Energy Sciences, USA

Mr. Paul H. Wright, Indianapolis, Indiana, USA 
The Princeton Plasma Physics Laboratory is operated by Princeton University under contract with the U.S. Department of Energy.

\author{
Information Services \\ Princeton Plasma Physics Laboratory \\ P.O. Box 451 \\ Princeton, NJ 08543
}

Phone: 609-243-2750

Fax: 609-243-2751

e-mail: pppl_info@pppl.gov

Internet Address: http://www.pppl.gov 